\title{
Comparison of Various Hybrid Image Enhancement Techniques
}

\author{
1.Neeru Malik, 2.Arun Rana \\ 1.Mtech student,Department of Electronics and communication,DVIET,karnal \\ 2.AssistantProfessor,Department of Electronics and communication,DVIET, Karnal
}

\begin{abstract}
Image Enhancement is simple and most appealing area among all the digital image processing techniques. The main purpose of image enhancement is to bring out detail that is hidden in an image or to increase contrast in a low contrast image. The principal objective of image enhancement is to modify attributes of an image to make it more suitable for a given task and a specific observer. During this process, one or more attributes of the image are modified. The choice of attributes and the way they are modified are specific to a given task. Moreover, observer-specific factors, such as the human visual. Whenever an image is converted from one form to other such as digitizing the image some form of degradation occurs at output. Improvement in quality of these degraded images can be achieved by using application of enhancement techniques. Various enhancement schemes are used for enhancing an image which includes gray scale manipulation, filtering and Histogram equalization. Histogram equalization is one of the well known image enhancement technique. In this paper comparative analysis of different enhancement techniques has been carried out. Dfferent parameter for each of the technique is also calculated.
\end{abstract}

Keywords: Contrast enhancement, Histogram equalization, PSNR, MSE NAE, CPSNR, Visual quality.

\section{INTODUCTION}

Image enhancement is among the simplest and most appealing areas of digital image processing Basically ,the idea behind enhancement techniques is to bring out detail that is obscured, or simply to highlight certain features of interest in an image. A familiar example of enhancement is shown in Fig. 1 in which when we increase the contrast of an image and filter it to remove the noise "it looks better." It is important to keep in mind that enhancement is a very subjective area of image processing. Improvement in quality of hese degraded images can be achieved by using application of enhancement techniques
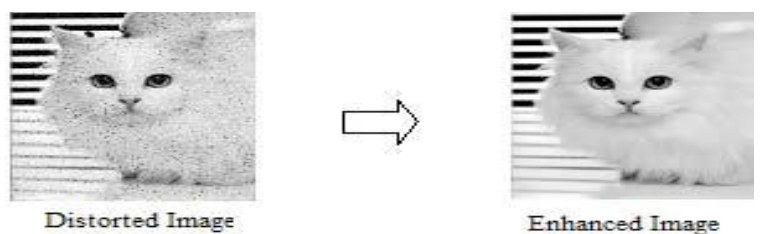

Fig.1: Image enhancement

\section{A. Global Histogram Equalization Method:}

Global image enhancement uses the histogram information of the entire input image for its transformation function. Though this global approach is suitable for overall enhancement, it fails to adapt with the local brightness features of the input image. If there are some gray levels in the image with very high frequencies, they dominate the other gray levels having lower frequencies. In such a situation, GHE remaps the gray levels in such a way that the contrast stretching becomes limited in some dominating gray levels having larger image histogram components and causes significant contrast loss for other small ones.

\section{B. Adaptive Histogram Equalization Method:}

This is an excellent contrast enhancement method for both natural images and medical and other initially non visual images. This mehod involves applying to each pixel the histogram equalization mapping based on the pixels in a region surrounded that pixel(its contextual region ). That is each pixel is mapped to an intensity proportional to its rank in the pixel surrounding it. But basic method is slow , and under certain conditions the enhanced image has undesirable features.

\section{Contrast Limited Histogram Equalization Method:}

This is an extension to traditional Histogram Equalization technique. It enhances the contrast of images by transforming the values in the intensity image I. Unlike HISTEQ, it operates on small data regions 
(tiles), rather than the entire image. Each tile's contrast is enhanced, so that the histogram of the output region approximately matches the specified histogram. The neighboring tiles are then combined using bilinear interpolation in order to eliminate artificially induced boundaries. The contrast, especially in homogeneous areas, can be limited in order to avoid amplifying the noise which might be present in the image.

\section{Dualistic Sub Image Histogram Equalisation Method:}

This is a novel histogram equalization technique in which the original image is decomposed into two equal area sub-images based on its gray level probability density function. Then the two sub-images are equalized respectively. At last, we get the result after the processed sub-images are composed into one image. In fact, the algorithm can not only enhance the image visual information effectively, but also constrain the original image's average luminance from great shift. This makes it possible to be utilized in video system directly.

\section{BACKGROUND}

One of the first applications of digital images was in the newspaper industry, when pictures were first sent by submarine cable between London and New York. Introduction of the Bartlane cable picture transmission system in the early 1920s reduced the time required to transport a picture across the Atlantic from more than a week to less than three hours. Specialized printing equipment coded pictures for cable transmission and then reconstructed them at the receiving end. Some of the initial problems [2] in improving the visual quality of these early digital pictures were related to the selection of printing procedures and the distribution of intensity levels .Although the methods just cited involve digital images, they are not considered digital image processing results in the context of our definition because computers were not involved in their creation. Thus, the history of digital image processing is intimately tied to the development of the digital computer.

\section{IMPLIMENTATION}

Compare all these techniques on the basis of performance parameters in objective and subjective manner. These are the merits on the bases of that I will compare above definedtechniques.

\section{A.Global Histogram Equalization Method:}

Algorithm steps:

1. Computing the Histogram

Parse the input image

$>$ Count each distinct pixel value in the image

$>$ Ex. for 8-bit pixels, the Max Pixel Value is 255, and array size is 256.

2. Computing the normalized sum of histogram

$>$ Store the sum of all the histogram values

$>$ normalize by multiplying each element by (maximum-pixel-value/number of pixels).

3. Transforming input image into output image

- Use the normalized array as a look up table for mapping the input image pixel value to the new set of values from stage

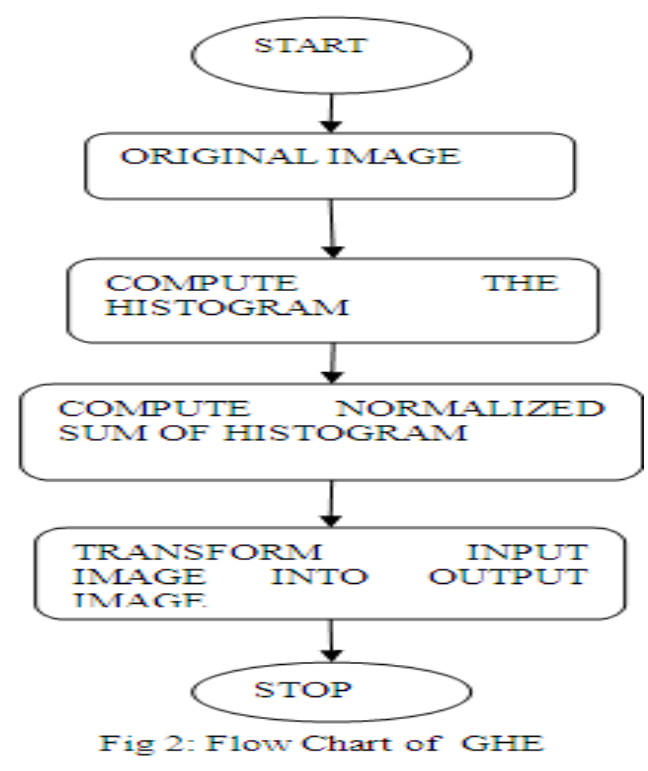




\section{B. Adaptive Histrogram Equalization Method:}

Algorithm steps:

1. Compute and apply mapping histogram by histrogram

2. Weight temporary images by modifying bilinear weighting functions, and sum results.

3. Fix up corners and borders.

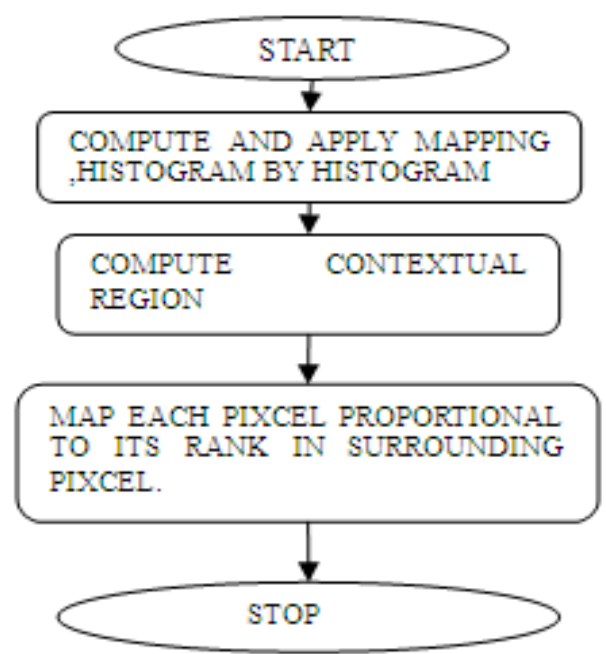

Fig3: Flow Chart of AHE.

\section{Contrast Limited Adaptive Histogram Equalization Method:}

Algorithm Steps:

1. Obtain all the inputs: Image, Number of regions in row and column directions, Number of bins for the histograms used in building image transform function (dynamic range), Clip limit for contrast limiting (normalized from 0 to 1 )

2. Pre-process the inputs: Determine real clip limit from the normalized value if necessary, pad

3. Process each contextual region (tile) thus producing gray level mappings: Extract a single image region, make a histogram for this region using the specified number of bins, clip the histogram using clip limit, create a mapping (transformation function) for this region

4. Interpolate gray level mappings in order to assemble final CLAHE image: Extract cluster of four neighbouring mapping functions, process image region partly overlapping each of th

e mapping tiles, extract a single pixel, apply four mappings to that pixel, and interpolate between the results to obtain the output pixel; repeat over the entire image.

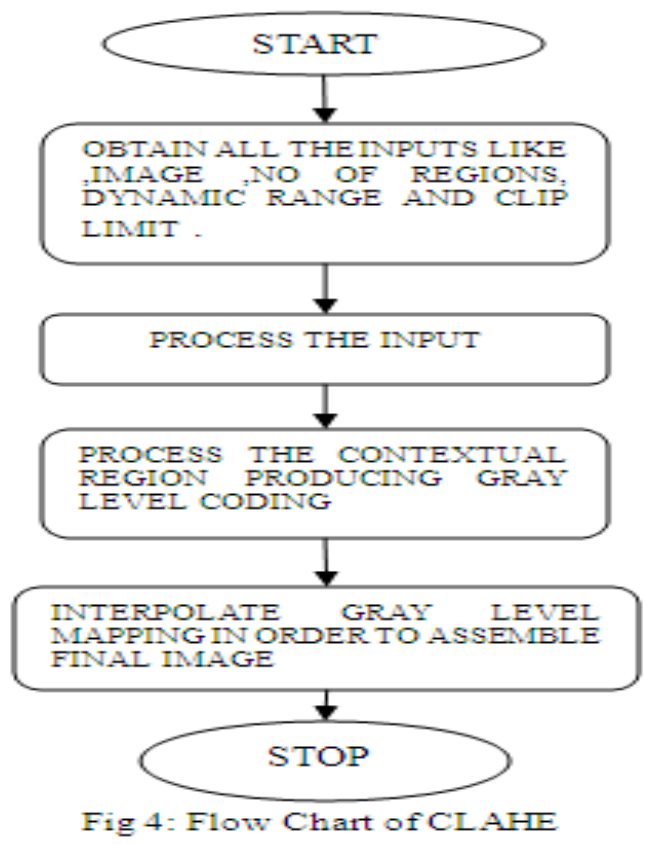




\section{Equal area Dualistic sub-image histogram equalization} method:

Algorithm Steps:

Suppose image $\mathrm{X}$ is segmented by a section with gray level of $\mathrm{X}=\mathrm{Xe}$ and the two sub-images are $\mathrm{Xl}$ and $\mathrm{Xu}$, so we have, $\mathrm{X}=\mathrm{XIUXU}$. Here $\quad \mathrm{XL}=(\mathrm{X}(\mathrm{i}, \mathrm{j}) / \mathrm{X}(\mathrm{i}, \mathrm{j})<\mathrm{Xe}, \forall \mathrm{X}(\mathrm{i}, \mathrm{j}) \in X)$ $(X(i, j) \backslash X(i, j) \geq X e, \forall X(i, j) \in X)$

It is obvious that sub image $\mathrm{XL}$ is composed by gray level of $\{\mathrm{X} 0, \mathrm{X} 1$,

$\mathrm{Xe}-1\}$, while sub image $\mathrm{XU}$ is composed of $\{\mathrm{Xe}, \mathrm{Xe}+1, \ldots \ldots \ldots \ldots . \mathrm{Xl}-1\}$. The aggregation of the original images' gray level distribution probability is decomposed into $\{\mathrm{p} 0, \mathrm{p} 1$

pe-1\} and $\{\mathrm{pe}, \mathrm{pe}+1, \ldots \ldots \ldots \ldots . . . \mathrm{pl}-1\}$ correspondingly. The corresponding cumulative distribution function will be

$$
\mathrm{CL}\left(\mathrm{x}_{\mathrm{k}}\right)=1 / \mathrm{p} \sum \mathrm{p}^{\mathrm{i}}, \mathrm{k}=0,1 \ldots \ldots \ldots \ldots \ldots \ldots . . \mathrm{e}-1
$$

$\mathrm{C}_{\mathrm{U}}\left(\mathrm{X}_{\mathrm{K}}\right)=1 / \mathrm{p}-1 \sum \quad \mathrm{p}_{\mathrm{i}}, \mathrm{k}=\mathrm{e}, \mathrm{e}+1 \ldots \ldots \ldots \ldots \ldots . \mathrm{L}-1$

Based on the cumulative distribution function, the transform functions of the two sub images' histogram are equalized below.

$$
\begin{gathered}
\mathrm{F}_{\mathrm{L}}\left(\mathrm{X}_{\mathrm{k}}\right)=\mathrm{X}_{0}+\left(\mathrm{X}_{\mathrm{e}}-1-\mathrm{X}_{0}\right) \mathrm{c}\left(\mathrm{X}_{\mathrm{k}}\right), \\
\mathrm{k}=0,1, \ldots \mathrm{e}-1 \ldots \\
\mathrm{F}_{\mathrm{U}}\left(\mathrm{X}_{\mathrm{k}}\right)=\mathrm{Xe}=\left(\mathrm{X}_{1}-1-\mathrm{Xe}\right) \mathrm{c}(\mathrm{Xk}), \\
\mathrm{k}=\mathrm{e}, \mathrm{e}+1, \ldots \ldots \ldots \ldots . \mathrm{L}-1
\end{gathered}
$$

At last result of dualistic sub image histogram is obtained after the two equalized sub images are composed into one image.

Suppose $\mathrm{Y}$ denotes the processed image then $\mathrm{Y}=\{\mathrm{Y}(\mathrm{i}, \mathrm{j})\}=\mathrm{f}_{\mathrm{L}}\left(\mathrm{X}_{\mathrm{L}}\right) \mathrm{U} \mathrm{f}_{\mathrm{U}}\left(\mathrm{X}_{\mathrm{U}}\right) \ldots$

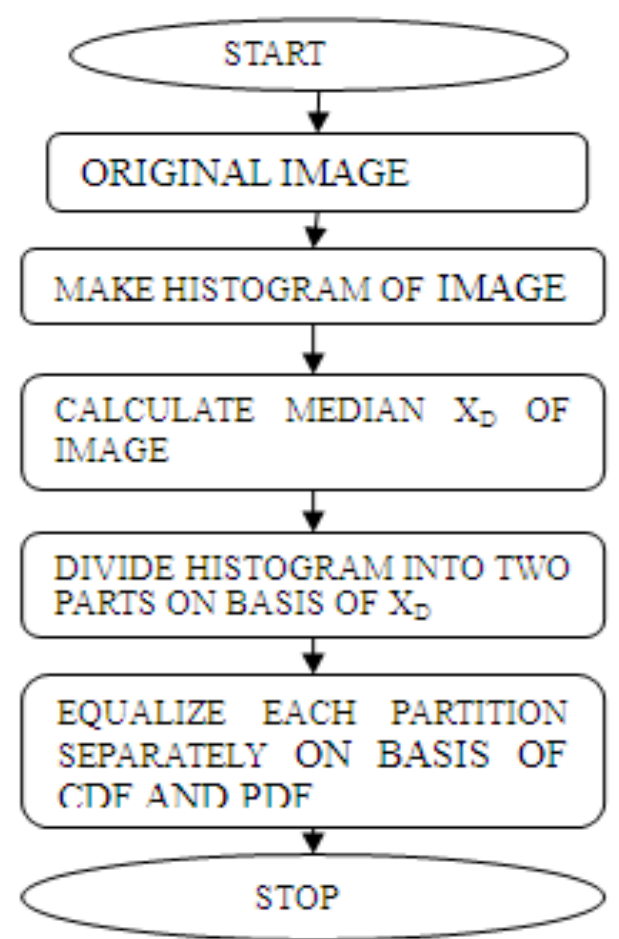

Fig 5: Flow chart of DSHIE

\section{E. Metrics for Gray Scale Images:}

\section{Peak-signal-to-noise-ratio (PSNR):}

PSNR is the evaluation standard of the reconstructed image quality, and is important measurement feature. PSNR is measured in decibels $(\mathrm{dB})$ and is given by:

\section{PSNR $=10 \log \left(255^{2} / \mathrm{MSE}\right)$}

where the value 255 is maximum possible value that can be attained by the image signal. Mean square error (MSE) is defined as Where $\mathrm{M}^{*} \mathrm{~N}$ is the size of the original image. Higher the PSNR value is, better the reconstructed image is. 
2. Absolute mean brightness error (AMBE):

It is the Difference between original and enhanced image and is given as

AMBE $=|\mathbf{E}(\mathbf{X})-\mathbf{E}(\mathbf{Y})|$

Where $\mathrm{E}(\mathrm{x})=$ average intensity of input image $\mathrm{E}(\mathrm{y})=$ average intensity of enhanced image

\section{Normalised absolute error:}

$$
N A E=\frac{\sum_{j=1}^{M} \sum_{k=1}^{N}|X(j, k)-\hat{X}(j, k)|}{\sum_{j=1}^{M} \sum_{k=1}^{N}|X(j, k)|}
$$

4. Contrast:

Contrast defines the difference between lowest and highest intensity level. Higher the value of contrast means more difference between lowest and highest intensity level.

\section{Visual Quality:}

By looking at the enhanced image, one can easily determine the difference between the input image and the enhanced image and hence, performance of the enhancement technique is evaluated.

\section{TOOL TO BE USED}

In this thesis for implementation of techniques MATLAB 7.6

version will be used. In that image processing toolbox is used. MATLAB ${ }^{\circledR}$ is a high-performance language for technical computing.

\section{EXPERIMENTAL RESULT}

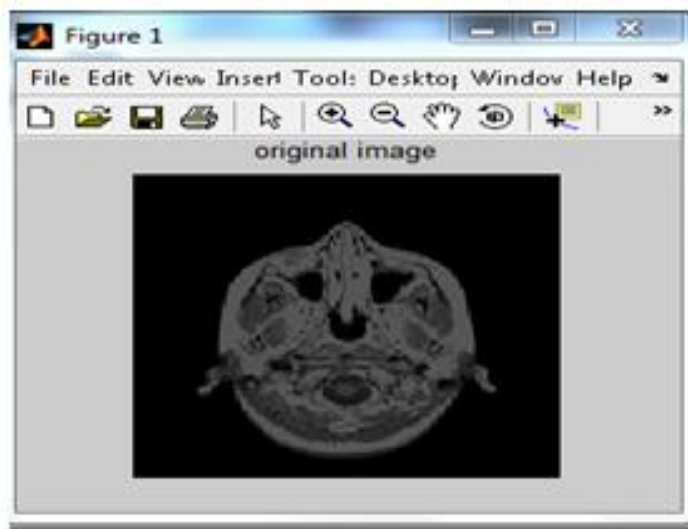

(a) Original image

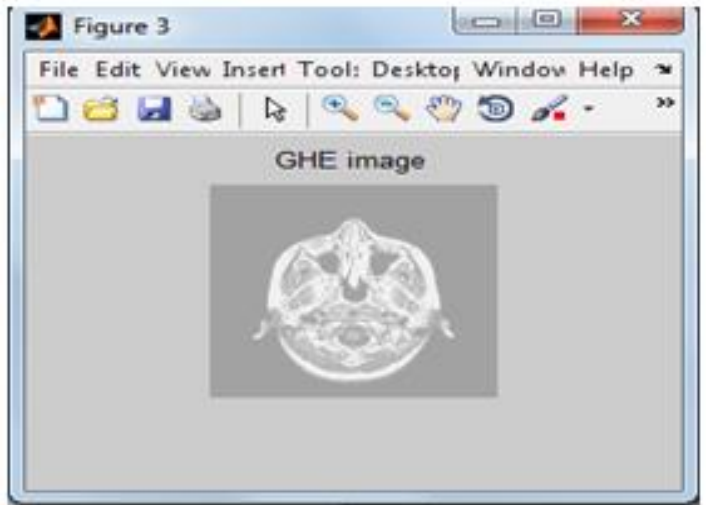

(C) GHE Image

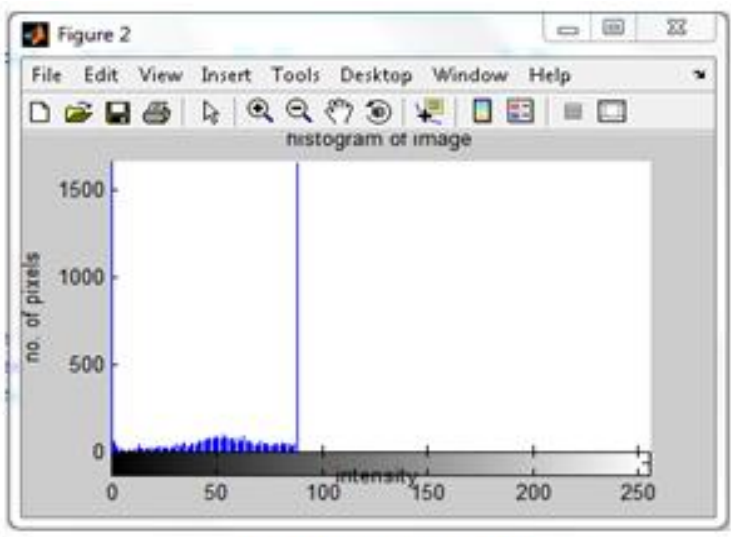

(b) Histogram of image

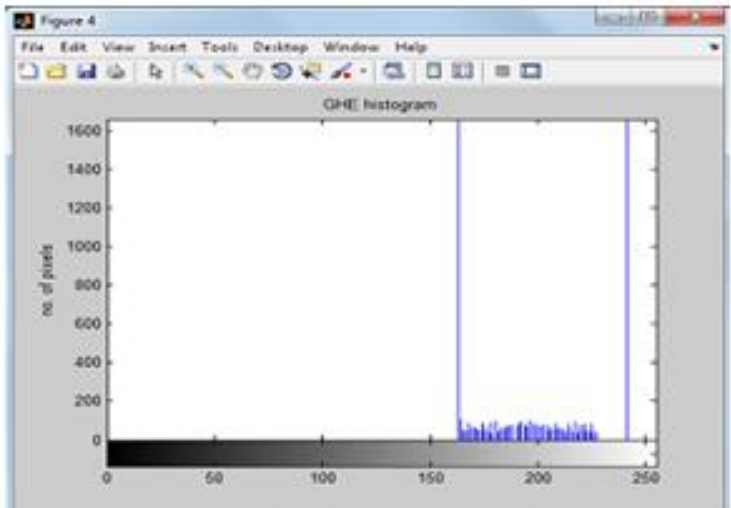

(d) GHE Histogram 


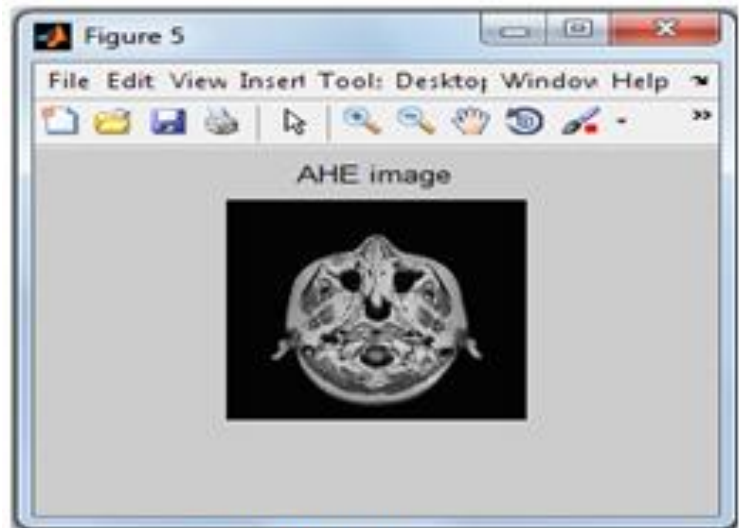

(e) AHE Image

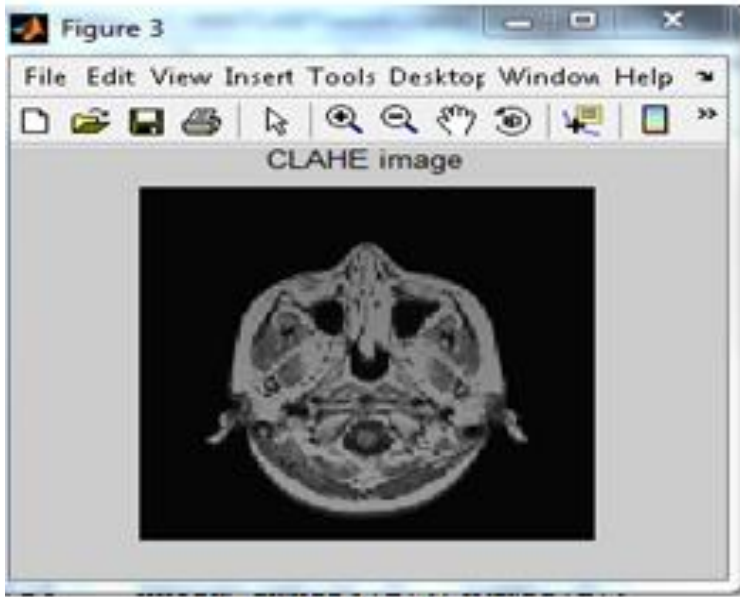

(g) CLAHE Image

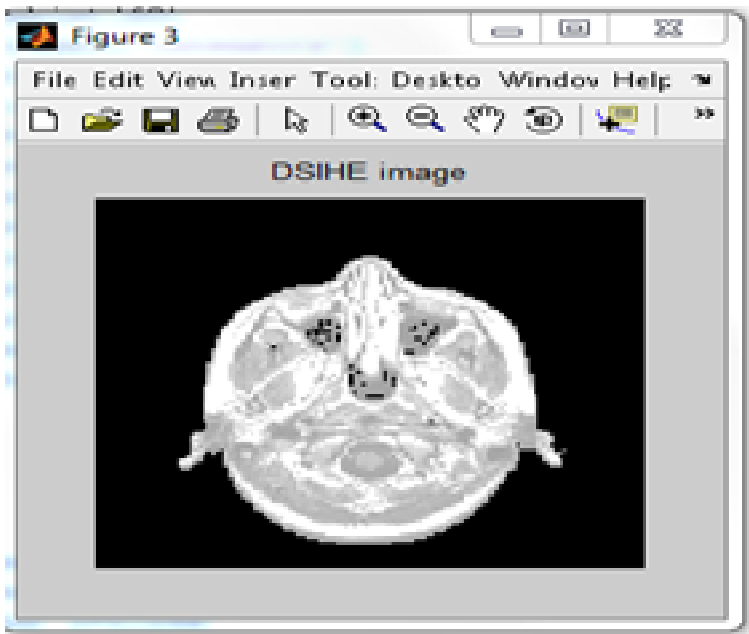

(i) DSLHE Image

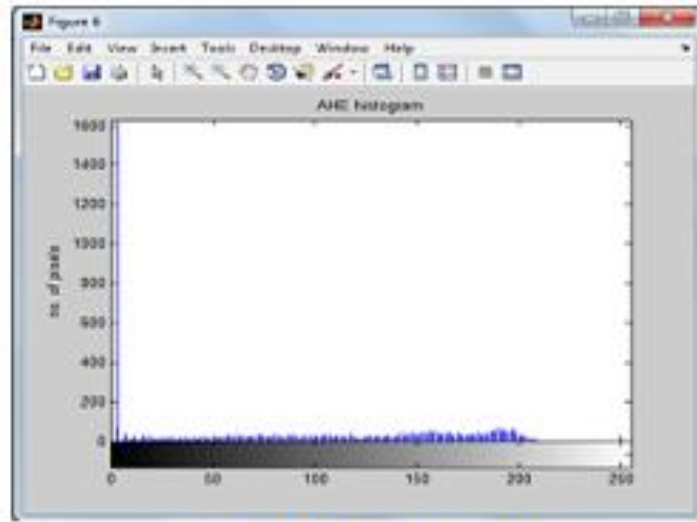

(f) AHE Histogram

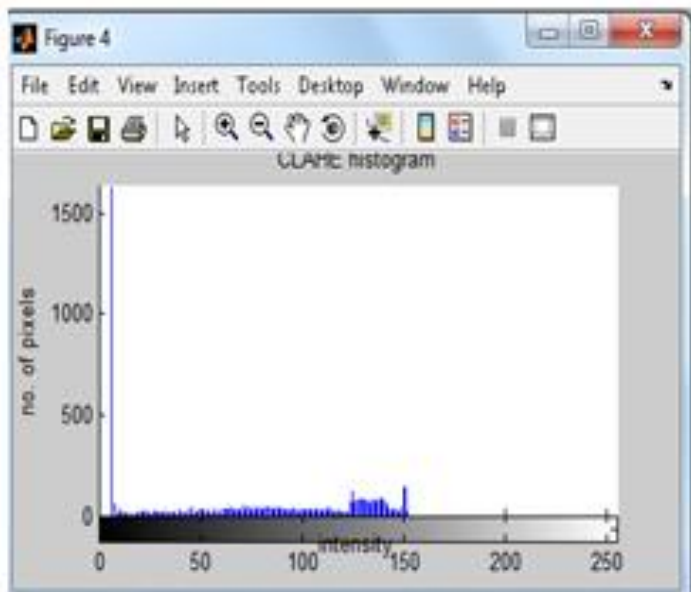

(h) CLAHE Histogram

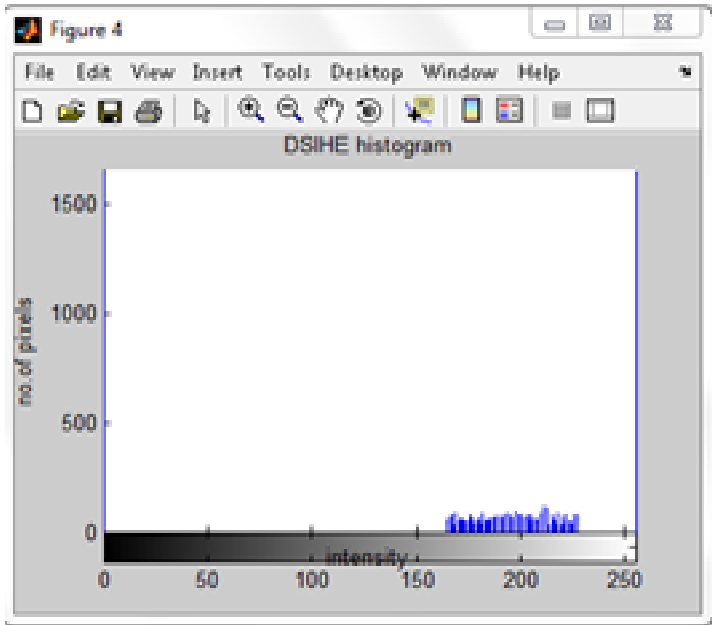

(j) DSIHE Image.

\begin{tabular}{|l|l|l|l|l|}
\hline PARAMETER & AMBE & CONTRAST & PSNR & NAE \\
\hline GHE & 157.3438 & 6.0952 & 2.0909 & 7.0424 \\
\hline AHE & 23.9788 & 32.5689 & 7.9352 & $\mathbf{1 . 0 7 4 4}$ \\
\hline CLAHE & $\mathbf{1 5 . 7 3 2 4}$ & 15.9878 & $\mathbf{2 0 . 9 7 2 1}$ & 0.7042 \\
\hline DSIHE & 55.1034 & $\mathbf{7 1 . 3 4 8 9}$ & 8.8654 & 2.4663 \\
\hline
\end{tabular}




\section{CONCLUSION AND FUTURE SCOPE}

In this Paper, a flow chart for image enhancement based on prior knowledge on the Histogram Equalization has been presented. Many image enhancement schemes like Global Histogram Equalization, Adaptive Histogram Equalisation, Contrast limited Adaptive Histogram Equalization (CLAHE), Equal area dualistic sub-image histogram equalization (DSIHE) has been implemented and compared. The Performance of all these Methods has analyzed by a number of Practical experiments of real time images .

In Future,different images can be taken and implemented for the given factors .here only grey scale images has used.but same techniques can be used for colour images.

\section{REFERENCES}

[1]. S. Lau, "Global image enhancemS. Lau, "Global image enhancement using local information," Electronics Letters, vol. 30, pp. 122-123, Jan. 1994.

[2]. J. Zimmerman, S. Pizer, E. Staab, E. Perry, W. McCartney, B. Brenton, "Evaluation of the effectiveness of adaptive histogram equalization for contrast enhancement," IEEE Transactions on Medical Imaging, pp. 304-312, 1988.

[3]. Yu Wan, Qian Chen, Bao-Min Zhang, "Image enhancement based on equal area dualistic sub-image histogram equalization method," IEEE Transactions Consumer Electron., vol. 45, no. 1, pp. 68-75, 1999.

[4]. Yeong-Taeg Kim, "Contrast enhancement using brightness preserving bi-histogram equalization," IEEE Trans. Consumer Electronics, vol. 43, no. 1, pp. 1-8, 1997.

[5]. M. Abdullah-Al-Wadud, Md. Hasanul Kabir, M. Ali Akber Dewan, Oksam Chae, "A dynamic histogram equalization for image contrast enhancement", IEEE Transactions. Consumer Electron., vol. 53, no. 2, pp. 593- 600, May2007.

[6]. Wang Yuanji. Li Jianhua, Lu E, FuYaoJiang Qinzhong, "Image Quality Evaluation Based On Image Weighted Separating Block Peak Signal To Noise Ratio", IEEE Int. Conf. Neural Networks \& Signal Processing, Nanjing, China, December 14-17, 2003.

[7]. Rafael C. Gonzalez, Richard E. Woods, "Digital Image Processing", $2^{\text {nd }}$ editionPrentice Hall, 2002.

[8]. Stephen M. Pizer, R. Eugene Johnston, James P. Ericksen, Bonnie C. Yankaskas, Keith E. Muller, "Contrast-Limited

[9]. Adaptive Histogram Equalization Speed and Effectiveness", ", IEEE Int. Conf. Neural Networks \& Signal Processing, Nanjing, China, December 14-17, 2003.

[10]. Rafael C. Gonzalez, Richard E. Woods, "Digital Image Processing", 2nd edition, Prentice Hall, 2002.

[11]. A. K. Jain, "Fundamentals of Digital Image Processing". Englewood Cliffs, NJ: Prentice-Hall, 1991.

[12]. Rajesh garg,Bhawana Mittal,Sheetal garg, "Histogram equalization techniques for image enhancements"IJECT.,VOL..2,March 2011. 